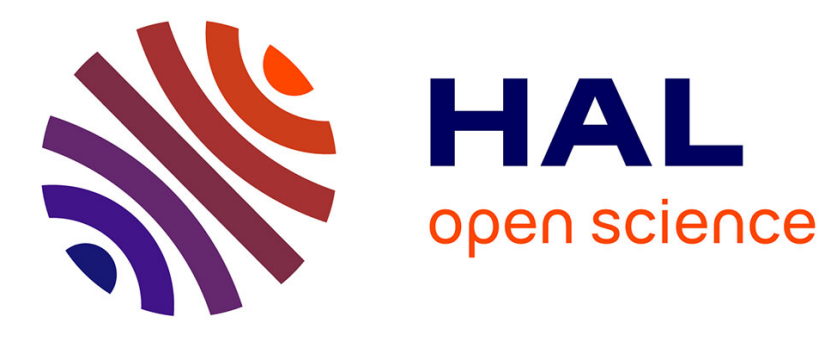

\title{
Hydroxy Channels-Adaptive Pathways for Selective Water Cluster Permeation
}

\author{
Li-Bo Huang, Arthur Hardiagon, Istvan Kocsis, Cristina-Alexandra Jegu, \\ Mihai Deleanu, Arnaud Gilles, Arie van Der Lee, Fabio Sterpone, Marc \\ Baaden, Mihail Barboiu
}

\section{To cite this version:}

Li-Bo Huang, Arthur Hardiagon, Istvan Kocsis, Cristina-Alexandra Jegu, Mihai Deleanu, et al.. Hydroxy Channels-Adaptive Pathways for Selective Water Cluster Permeation. Journal of the American Chemical Society, 2021, 143 (11), pp.4224-4233. 10.1021/jacs.0c11952 . hal-03364954v2

\section{HAL Id: hal-03364954 https://hal.science/hal-03364954v2}

Submitted on 31 Oct 2021

HAL is a multi-disciplinary open access archive for the deposit and dissemination of scientific research documents, whether they are published or not. The documents may come from teaching and research institutions in France or abroad, or from public or private research centers.
L'archive ouverte pluridisciplinaire HAL, est destinée au dépôt et à la diffusion de documents scientifiques de niveau recherche, publiés ou non, émanant des établissements d'enseignement et de recherche français ou étrangers, des laboratoires publics ou privés. 


\title{
Hydroxy-channels - adaptive pathways for highly selective water cluster permeation
}

\author{
Li-Bo Huang, ${ }^{\dagger}+$ Arthur Hardiagon,,${ }^{\#}$ Istvan Kocsis, ${ }^{\ddagger}{ }^{\mathbb{I}}$ Cristina-Alexandra Jegu, ${ }^{\ddagger}$ Mihai Deleanu, ${ }^{\ddagger}$ Ar- \\ naud Gilles, ${ }^{*}$ Arie van der Lee, ${ }^{\ddagger}$ Fabio Sterpone, ${ }^{\#, ~ M a r c ~ B a a d e n, ~}{ }^{\#, \S}$ and Mihail Barboiu*,†, \\ ${ }^{\dagger}$ Lehn Institute of Functional Materials, School of Chemistry, Sun Yat-Sen University, Guangzhou 510275, China \\ *Institut Europeen des Membranes, Adaptive Supramolecular Nanosystems Group, University of Montpellier, ENSCM- \\ CNRS, UMR5635, Place E. Bataillon CC047, 34095 Montpellier, France \\ ${ }^{\#}$ CNRS, Université de Paris, UPR 9080, Laboratoire de Biochimie Théorique, 13 rue Pierre et Marie Curie, F-75005, Paris, \\ France
}

$\S$ Institut de Biologie Physico-Chimique-Fondation Edmond de Rotschild, PSL Research University, Paris, France

\begin{abstract}
Artificial water channels-AWCs are known to selectively transport water, with ion exclusion. Similar to natural porins, AWCs encapsulate water wires or clusters, offering continuous and iterative H-bonding that play a vital role in their stabilization. Herein, we report octyl-ureido-polyol AWCs capable of self-assembly into hydrophilic hydroxy-channels. Variants of ethanol, propanediol and trimethanol are used as headgroups to modulate water transport permeabilities, with complete rejection of ions. The hydroxy-channels achieve a single-channel permeability of $2.33 \times 10^{8}$ water molecules per second, which is within the same order of magnitude as the transport rates for Aquaporins. Depending on their concentration in the membrane, adaptive channels are observed in the membrane. Over increased concentrations, a significant shift occurs initiating unexpected higher water permeation. Molecular simulations probe that sponge-like or cylindrical aggregates can form to generate transient cluster water pathways through the bilayer. Altogether, the adaptive self-assembly is a key feature influencing channel efficiency The adaptive channels described here may be considered as an important milestone contributing to the systematic discovery of artificial water channels for water desalination.
\end{abstract}

\section{INTRODUCTION}

One of the most ambitious goals in separation sciences today, is to accomplish the reconstruction of natural carriers or channels through synthetic design. Proteins have evolved for millennia to adopt their current functional structures. Although they are remarkable in functional efficacy, their structural complexity and environmental sensibility undermine their reproducibility and further applications for "out of the membrane cell" use. ${ }^{1}$

A key step in achieving this goal is the replacement of natural transporters with simpler synthetic analogues. Synthetic biomimetic approaches, offer us the possibility to build artificial unimolecular or supramolecular constructs that can mimic biological functionality, while featuring procedural and structural simplicity. ${ }^{2}$ The functions of carriers, ion-channels and porins have been successfully replicated using small building blocks. There are several advantages for using artificial mimics; most notable are the possible lower costs, tunability, improved stability and compatibility with the membrane host matrices.

After a decade of their discovery, ${ }^{3-7}$ Artificial water channels - AWCs come very close to the performance of the natural counterparts, the Aquaporins. Carbon Nanotube PorinsCNTPs (Figure 1a,b), ${ }^{8,9}$ Pillar[5] arene-PAP5 (Figure 1c) ${ }^{10,11}$ Pillar[4] arene-PAH[4] clusters (Figure 1d), ${ }^{12}$ Aquafoldamers (Figure 1e), ${ }^{13}$ Porous organic cages-POC (Figure 1f), ${ }^{14}$ Pillar[5] arene Hydrazones-PAH5 ${ }^{7}$ and Pillar[5]arene-AQPs ${ }^{15}$ are able to close- or even out-perform AQPs, when it comes to water permeability and/or selectivity. ${ }^{1}$ In all artificial setups, transport of water occurs either through a single water file, as seen in the case of AQPs, or through the water cluster structures inside or outside the cavity of the channels. ${ }^{16}$

Increasing water-salt permselectivity or improving the salt rejection is the actual challenge to boost the efficiency of AWCs. ${ }^{16}$ Most artificial channels lack the complex mechanism involved in selective water transport, including wettingdewetting events, intermolecular water-water or channel-water $\mathrm{H}$-bonding, all these translated to water translocation through the channels. Only a few synthetic channels (I-quartet, PAH[4], POC, Aquafoldamers, Pillar[5]arene -AQPs) are capable of selective rejection of ions. In these examples, the water transport takes places via water wires or clusters. ${ }^{1,16}$

Structural issues with channels, dimensionally adapting the formation of wires or clusters, depending on their interior pore diameter, can enhance/reduce the water permeability that can significantly impede/exceed the channel selectivity. Weak interactions of water molecules with the hydrophobic walls of channels contribute to its nearly frictionless transport, while selectivity is sterically controlled. Structural design represents a formidable challenge in synthetic chemistry of AWCs. Within this context our pioneering work on I-quartet ${ }^{4,17}$ (Figure 1i) or I-quartet sponges ${ }^{18}$ (Figure $1 \mathrm{j}$ ) highlights the tremendous importance of controlling the water superstructures via channel-water $\mathrm{H}$-bonding events between communicating water molecules in the channel cavity, exerting the prohibition of ion insertion/translocation events. Obtaining absolute water selectivity resides in designing a channel, at least in its selectivity 
filter/region, with an environment that is favorable for the selective recognition/self-assembly of water via synergetic donor-acceptor H-bonding.

Basically, one molecule of water is able to form four hydrogen bonds, simultaneously acting as donor and acceptor through two bonds for each. We previously observed near perfect single water-wire formation within I-quartet channels, ${ }^{4,17}$ where the inner walls of the channels interact with a water molecule through one donor and one acceptor hydrogen bond, leaving the two other $\mathrm{H}$-bonds to be used to interconnect the water wires throughout the channel. Using the same principles, highly specific unimolecular water channels have been achieved using Aquafoldamers ${ }^{13}$ or Pillar[5]arene-AQPs ${ }^{15}$ in which water wires are stabilized via channel donor-acceptor $\mathrm{H}$-bonding, while having a lower performance than the AQPs and CNTP. ${ }^{2,3}$

Increasing the water-water $\mathrm{H}$-bonding results in the formation of clusters, that become highly selective in interactions with other species that are mostly rejected as they perturb the stability of water superstructures. Within this context, the concept of engineering cluster/sponge-type AWCs is clearly evolving, as it considerably expands the range of possibilities for the design of macroscopic biomimetic membrane materials embedding AWCs for reverse osmosis desalination. ${ }^{19}$

Combined with our previous experience in constructing self-assembled AWCs, ${ }^{17,18}$ herein we report a new class of AWCs based on octyl-ureido-polyols H1-H6 (Scheme 1) bearing hydrophilic hydroxyl groups, that form adaptive channels in lipid bilayers. Accommodating single water-wires or water-clusters, the channels are able to selectively transport water across a lipid membrane. We argue that such selective transport occurs through a selective water channel or waterwater pore assisted diffusion mechanism, controlled by donoracceptor hydroxyl bonding groups substantially influencing their permselectivity.

This scheme underlines the functional versatility of the artificial $\mathrm{OH}$-channels, containing the polar hydroxyl heads reminiscent to ceramide phospholipids that are now well-known for more than 40 years to be involved in cell regulation (apoptosis of cancer cells, differentiation...). ${ }^{20,21}$ Beside their cell regulation properties, ceramides are also known, when mixed with phospholipids, to generate two main effects: they increase the molecular order and they give rise to lateral phase and domain formation. Indeed, increase of the membrane permeability has been studied by Colombini et al. that have demonstrated the formation of pores. ${ }^{22}$ To the best of our knowledge, ceramides are the only class of non-proteogenic natural compounds able to form supramolecular assemblies that span the membrane bilayer, to generate pores for facilitated translocation.
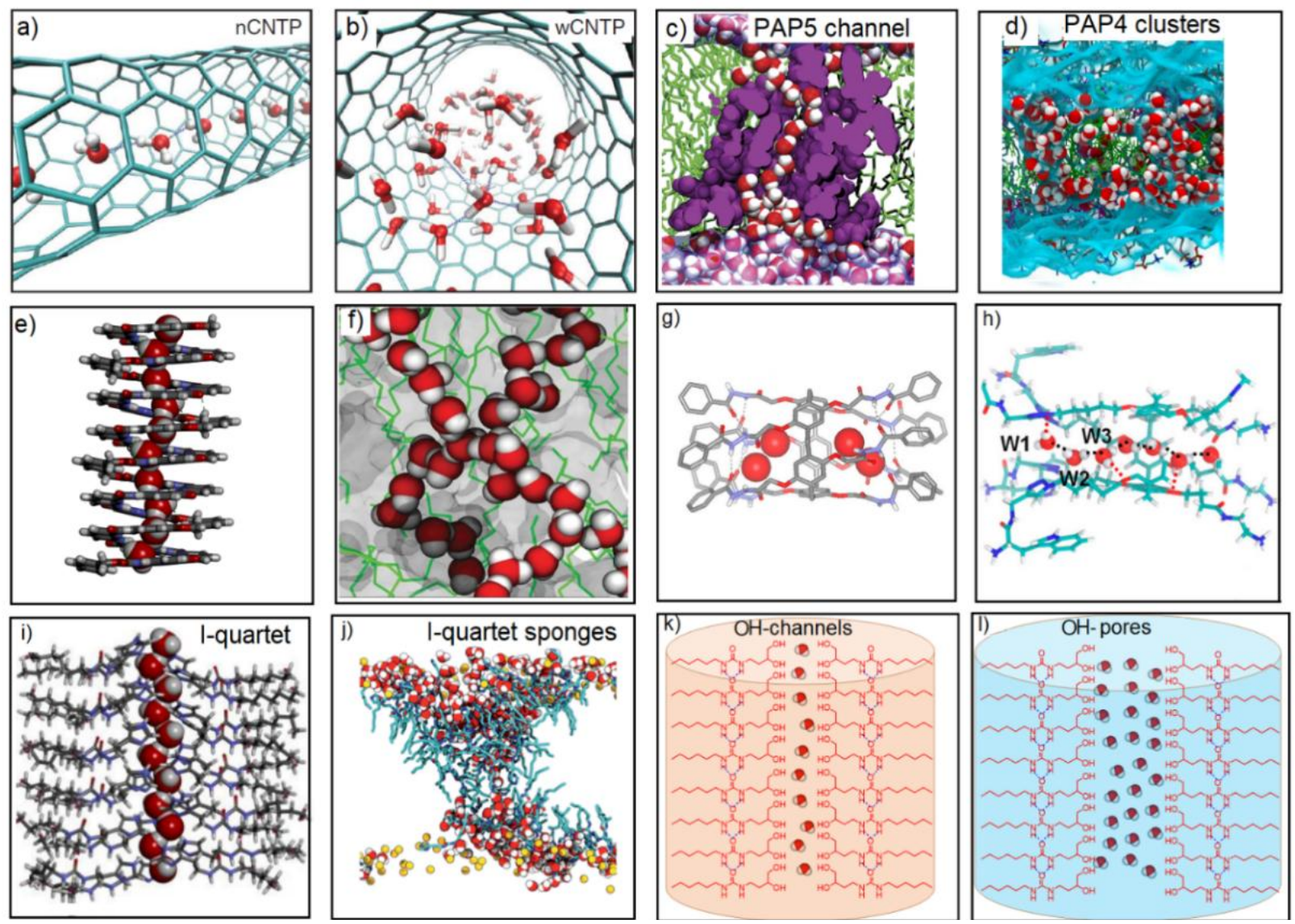

Figure 1. Artificial water channels. a) Narrow $n$ CNTPs and b) wider $w C N T P s$ Carbon Nanotubes Porins encapsulating single water-wires and water clusters $;{ }^{8}$ c) Pillar[5] arene-PAP5 channels for water-wires transport; ${ }^{11}$ d) water permeation through PAH[4] clusters in bilayer membrane $;^{12}$ e) single water-wire permeation through highly selective Aquafoldamers, ${ }^{13}$ f) water chains/clusters within POC nanoaggregates embedded in bilayer membrane $;{ }^{14} \mathrm{~g}$ ) encapsulated water dimers within Pillar[5]arene Hydrazones-PAH5; ${ }^{7}$ h) Molecular struc- 
ture of Pillar[5]arene-AQPs ${ }^{15}$ and its interaction with the single water-wire file; i) I quartet water channels and their interaction with single water wires $;{ }^{17} \mathrm{j}$ ) water clusters within I-quartet sponges embedded in bilayer membranes; ${ }^{18}$ adaptive $\mathrm{k}$ ) narrow $\mathrm{OH}$-channels and 1 ) wider $\mathrm{OH}$-pores described in this work, presenting hydroxylic decorated pathways for water-wires and water cluster translocation, through directional O- channels or OH-pores in bilayer membranes.

\section{RESULTS AND DISCUSSION}

Design Strategy. Herein, we focused on using simple octylureido-polyols building blocks H1-H6 (Scheme 1), that would be capable of combining alkyl ureido groups of the I-quartet and polyhydroxyl heads of natural ceramide channels. Therefore, we synthesized a series of compounds containing water/ion hydroxylic interacting heads and bearing an octyl chain in order to introduce optimal lipophilicity. ${ }^{17}$ The connection between these two elements is one urea group, known to form directional ribbon-type superstructures. ${ }^{18}$ Hydroxyl, as the simplest organic group for forming both donor and acceptor hydrogen bonds, has a natural affinity with water molecules. Ethanol, propanediol and trimethanol were employed as the hydrophilic binding motif for obtaining selective AWCs. Similar to the imidazole groups in I-quartet ${ }^{17,18}$ the "amphoteric" hydroxyl groups can selectively bind the water molecules through the donor-acceptor H-bonds.

Six compounds were synthetized for the studies presented here (Scheme 1). They were obtained through a one-step reaction between octyl-isocyanate and different amino-alkylpolyols in a mixture of solvents. The ${ }^{1} \mathrm{H}-,{ }^{13} \mathrm{C}$-NMR and ESIMS spectra agree with the proposed formulas (Figure S1-S18). The potential to form $\mathrm{OH}$-channel superstructures was assessed initially by crystallization of compounds H1-H6. X-ray single-crystal structures give a good indication of the self- assembly preferences of the compounds in solution and their possible self-organization in bilayer membranes. However, these assumptions may be treated with caution, as the solidstate single-crystal structures are representing only snapshots of possible arrangements of functional channels or pore forming aggregates in the bilayer membrane.

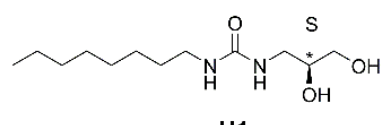

H1
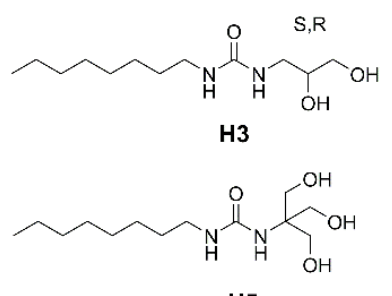

H5
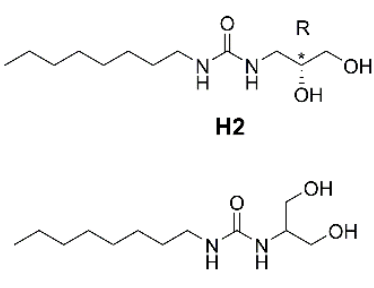

H4

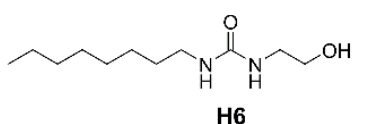

Scheme 1. Compounds H1-H6, as components for self-assembled hydroxy OH-Channels. 
a)

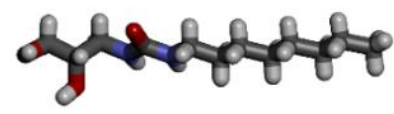

H1

b)

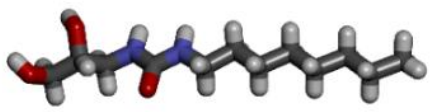

$\mathrm{H} 2$

c)

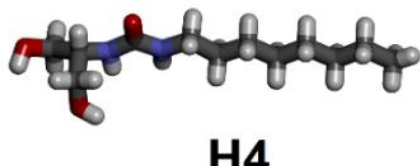

d)

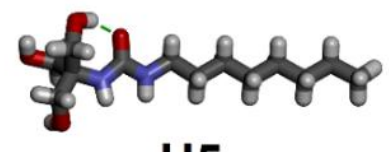

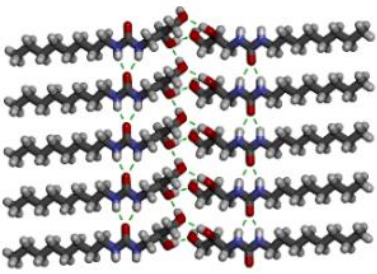
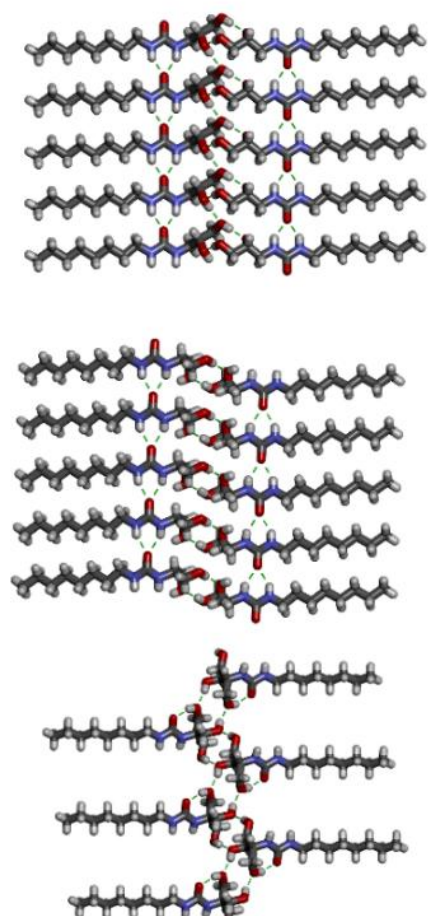
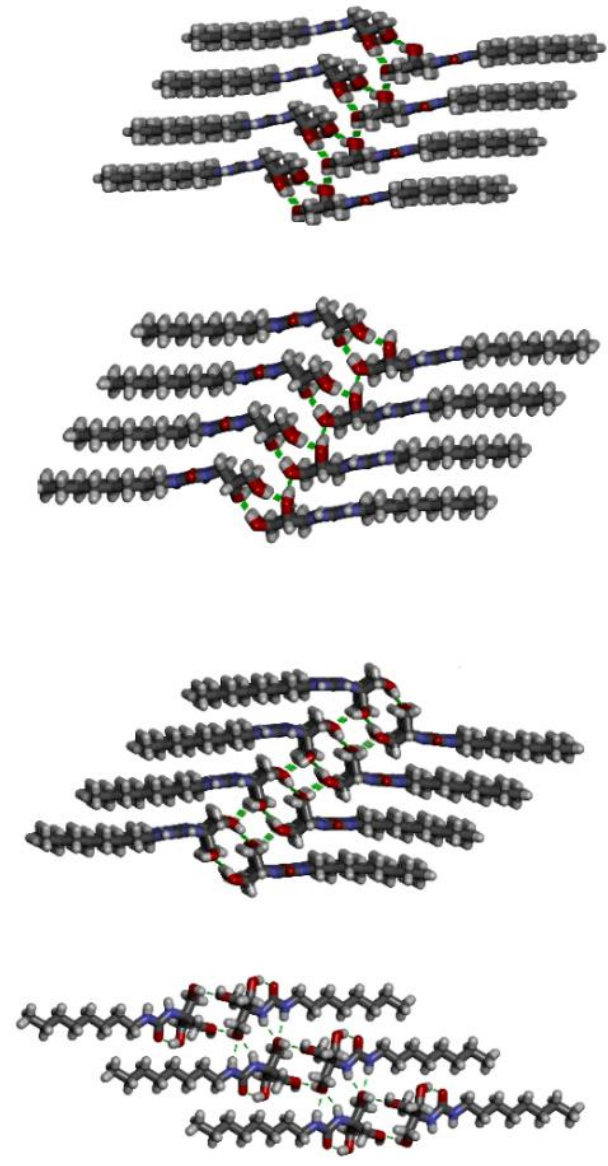

Figure 2. Crystal structures in stick representation of: a) H1, b) H2, c) $\mathbf{H 4}$ and d) $\mathbf{H 5}$ (N, blue; C, gray; O, red; H, white) showing (left) the monomeric molecules together with the lateral packing of H-bonded hydroxyl tetrads (middle) and their perpendicular packing via ureaurea and urea-hydroxyl H-bonding ribbons (right).

Single-crystals of good quality for X-ray structure determination were obtained for 1,2 diols $\mathbf{H 1}$ and $\mathbf{H 2}, 1,3$ diol $\mathbf{H 4}$ and 1,3,5 triol $\mathbf{H 5}$ (Table S1). Their solid-state structures reveal the formation of networks of $(\mathrm{OH}---\mathrm{O})_{4} \mathrm{H}$-bonding quartets, with the participation of two (H1, H2 and H5) and four (H4) different molecules each involving two or one hydroxyl arms, respectively. They form almost perfect rectangular assemblies with the sides of $\sim 2.7 \AA$. This $\mathrm{OH}$ tetrameric motif, then repeats both perpendicularly, as quartet component molecules are stacking through the urea moiety in crystal structures of H1, H2, and H4, as well as laterally, the second arm of the compounds participating in the formation of another adjacent $\mathrm{OH}$-quartet. The distance between laterally repeating units is $\sim 3.5 \AA$ (Figure 2a-c). Further MD simulation show that $\mathrm{OH}$ quartets are key motifs for the strong stabilization of the low impermeable water channels but they are not important for water translocation, while the losing the structural crystallinity of the channels in the membranes is an essential step of making these channels highly permeable, as previously observed with I-quartet sponges. ${ }^{18}$ The double H-bonding between the third HO-group and adjacent HNCONH urea groups disrupts the formation of urea ribbons, but preserves a strong perpendicular $\mathrm{H}$-bonding between interconnected $\mathrm{OH}$-quartets in the crystal structure of H5 (Figure 2d). Figures S19-S22 in Supporting information present structural details and CCDC
2015548-2015551 contains the supplementary crystallographic data for this paper.

Bilayer membrane transport experiments. In order to determine the transport permeability and selectivity, the $\mathrm{OH}$ channels H1-H6 were tested for water and ion transport experiments. Water transport was quantified through a scattering modulation method of a vesicle suspension using a stoppedflow setup, ${ }^{17}$ registering the shrinkage rate of vesicles under osmotic pressure to determine the induced osmotic water permeability of the $\mathrm{OH}$-channels, in the presence of $\mathrm{D}(+)$ sucrose or $\mathrm{NaCl}$, as competitive osmolytes (Figure 2). To determine if the compounds are also inducing ion transport, we conducted $\mathrm{pH}$ sensitive HPTS fluorometric experiments for different cations $\left(\mathrm{Li}^{+}, \mathrm{Na}^{+}, \mathrm{K}^{+}, \mathrm{Rb}^{+}\right.$and $\left.\mathrm{Cs}^{+}\right)$and anions $\left(\mathrm{Br}^{-}, \mathrm{I}^{-}, \mathrm{HCO}_{3}{ }^{-}, \mathrm{CO}_{3}{ }^{2-}, \mathrm{NO}_{3}{ }^{-}\right.$and $\mathrm{SO}_{4}{ }^{2-}$ ) and following the flow of species through the $\mathrm{OH}$-channels. By using control FCCP and Valinomycin as an assisting $\mathrm{H}^{+}$and $\mathrm{K}^{+}$carrier, respectively, we were able to check the specificity of the $\mathrm{OH}$-channels to transport ions and to determine the intrinsic selectivity of the channels, based on deconvoluted transport rates for cations and anions separately, rather focusing on their proton transport behaviors that could kinetically influence the translocation process. The transmembrane experiments offered all the prerequisites to assume that the compounds H1-H6 assemble into 
functional and highly selective water-permeable $\mathrm{OH}$-channels in liposomes.

Water-Transport Experiments. The $\mathrm{OH}$-channels were reconstituted into phosphatidylcholine (PC) / phosphatidylserine (PS) / Cholesterol (Chl), PC/PS/Chl mixture with a molar ratio of 4/1/5 lipid vesicles (100 nm in diameter). The channel-forming compounds were added by simple addition of the dimethyl sulfoxide DMSO solutions of H1-H6 to pre-formed vesicle suspensions using different compound to lipid molar ratios-mCLRs (see Supporting information for details). Then, the vesicles were exposed to outwardly directed osmotic pressure gradients. Under hypertonic conditions driven by outwardly $10 \mathrm{mM}$ PBS buffer solution $(\mathrm{pH}=7.4)$ of 115 or $200 \mathrm{mM} \mathrm{NaCl}$ or $200 \mathrm{mM} \mathrm{D(+)-Sucrose} \mathrm{osmolytes} \mathrm{(Figure}$ 3 , S24, S25), the shrinkage of the liposomes increased the light-scattering signal (Figure 2a). The water transport rates are strongly dependent on the concentration of the hydroxychannels, that increase the overall water permeability relative to the background lipid permeability in the bilayer membrane. The net permeabilities show a continual increase when mCLRs increase. A large range of permeabilities from 9.36 $\mu \mathrm{m} / \mathrm{s}$ to $19.63 \mu \mathrm{m} / \mathrm{s}$ can be obtained at mCLR $<1.48$ for H1H6. Interestingly for $\mathbf{H 2}, \mathbf{H 3}$, and $\mathbf{H 4}$ we observe an abrupt 10 -fold increase in water permeability values (160 to 560 $\mu \mathrm{m} / \mathrm{s}$ ) for mCLR > 1.48 (Figure S24, S25), reminiscent with the formation of larger channels/pores within the membrane. This unique adaptive behavior is probably related to an important concentration dependent morphology change of more permeable channels presenting different self-assembly behaviors within the membrane. To confirm this hypothesis the water transport measurements (Figure 3) were performed under different hypertonic solutions containing competitive osmolytes $\mathrm{D}(+)$-sucrose (Figure $\mathrm{S} 24$ ) or $\mathrm{NaCl}$ (Figure $\mathrm{S} 25$ ) covering low and high channel concentrations domains.
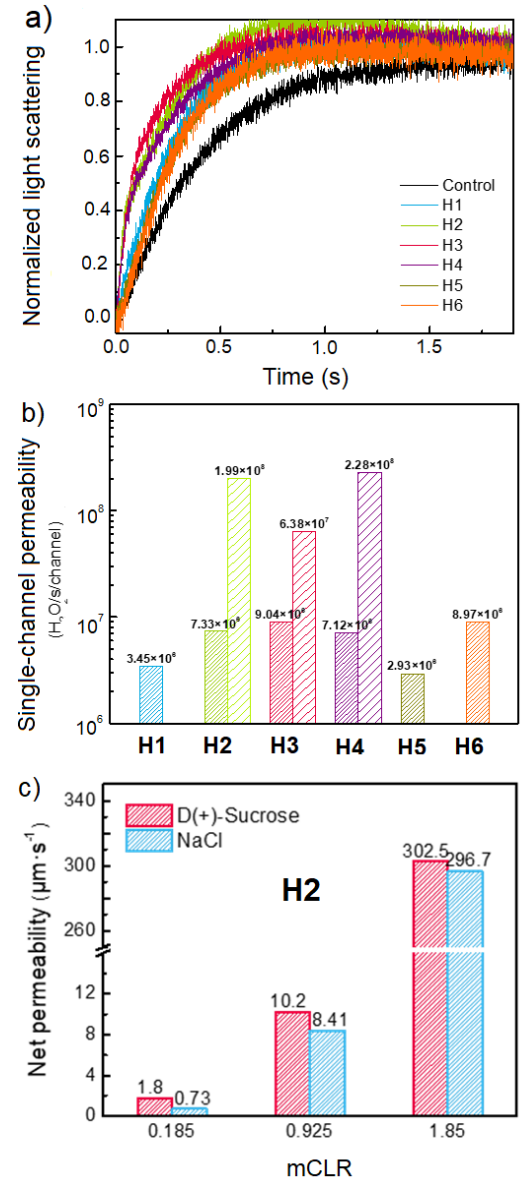

Figure 3. Measurements of water permeability through $\mathrm{OH}$ channels. a) Stopped-flow light scattering traces of liposomes containing channels at different $\mathrm{mCLR}=1.85,1.67,1.85,1.67$, 1.85 and 1.67, respectively; b) Single channel permeability calculated from the light scattering traces at different concentration in the membrane; c) The comparison of net permeability values for channels H2, determined by stopped-flow light scattering experiments with $200 \mathrm{mM} \mathrm{D}(+)$-Sucrose (pink) and $115 \mathrm{mM} \mathrm{NaCl}$ (blue) as osmolytes $\left(\Delta_{\text {osm }}=212 \quad \mathrm{mOsmol} / \mathrm{kg}\right)$.

hinder the hydrophilic hydroxyl groups to remain accessible to water. The situation is completely opposite for $\mathbf{H} 2$ and $\mathbf{H} 4$ showing higher water to salt selectivity at high concentration

We then performed carboxyfluorescein $(\mathrm{CF})$ leakage experiments ${ }^{23}$ to monitor channel formation at low and high concentration of H1-H6 (Figure S27). The magnitude of CF release is clearly different and the activity is increased in the following order: H4 $>\mathbf{H 3}>\mathbf{H 6}>\mathbf{H 1}>\mathbf{H 2}=\mathbf{H 5}$. According to these $\mathrm{CF}$ leakage experiments, it is possible to say that, at a high concentration for H4, H3 and unexpectedly for H6, there is the formation of large pores that allow efflux of $\mathrm{CF}$, while no activity was observed for H1, H2, H5. Colombini et al. ${ }^{22}$ observed the same behavior with ceramide, showing experimentally that the average channel size increased with the increase of ceramide content in the membrane. 
Table 1. Single-channel Permeability, $P_{s}$ of H1-H6 channels

\begin{tabular}{|c|c|}
\hline OH-Channel & $\mathrm{P}_{\mathrm{s}, \text { water molecules/s }}$ \\
\hline H1 & $3.54 \times 10^{6}$ \\
\hline H2 & $2.03 \times 10^{8}$ \\
\hline H3 & $6.84 \times 10^{7}$ \\
\hline H4 & $2.33 \times 10^{8}$ \\
\hline H5 & $4.89 \times 10^{6}$ \\
\hline H6 & $4.75 \times 10^{6}$ \\
\hline
\end{tabular}

We further estimated the single-channel permeability, $\mathrm{P}_{s}$ of H1-H6, based on the OH-channel insertion, lipid concentration and channel configuration in lipids (Table 1$)^{18}$ In order to determine the single-channel permeabilities, we used two rational assumptions: i) spontaneous total insertion of the compounds into the lipid bilayer. The total partitioning coefficients of H1-H6 in the bilayer membrane are confirmed as at maximal concentration of $80 \mathrm{mM}$ solutions are perfectly clear in the presence of lipid vesicles, while precipitate turbidity is formed when the compounds are added to aqueous solutions, reminiscent with almost total partition of H1-H6 in the bilayer membrane (please note that lower partition coefficients would lead to higher single channel permeabilities than those considered for total partition and ii) number of molecules needed to form a channel that spans the bilayer equal to the number of molecules found in the single crystal structure that correspond to 8 stacks of H1-H6 and 64 molecules per assembly within a transmembrane length of $5 \mathrm{~nm}$. Accordingly, the single channel permeability values varied obviously, covering the range of $3.5 \times 10^{6}-2.4 \times 10^{8}$ water/s/channel at CLR 0.026, which are only one orders of magnitude lower than that of AQPs $\left(\sim 10^{8}-10^{9}\right.$ water $/ \mathrm{s} /$ channel $\left.^{1,2}\right)$ (Figure 3 , Table 1$)$. Water transport activities increase substantially at higher concentrations of H2-H4, implying the formation of highly permeable channels. Indeed, the hydrophilic diol heads seemed to be more optimized for permeation than monol and triol heads, leading to higher water permeation when mixed with lipids. The tendency for increase of net and single channel permeability at high concentration can be explained by progressive higher cluster-type aggregation of $\mathrm{OH}$-channels, promoting the increased water transport via sponge like structures as we previously observed for I-quartet channels. ${ }^{18}$ Differently, a low concentration of $\mathrm{OH}$-channels prevents the aggregation and allows only reduced water-wires translocation.

Cation and Anion Transport Experiments. Cation $\left(\mathrm{M}^{+}=\right.$ $\mathrm{Li}^{+} \mathrm{Na}^{+}, \mathrm{K}^{+}, \mathrm{Rb}^{+}$and $\left.\mathrm{Cs}^{+}\right)$transport activities across the bilayer membranes incorporating H1-H6 OH-channels, reconstituted into phosphatidylcholine (PC) lipid vesicles $(100 \mathrm{~nm})$, at the same mCLRs as used for the water transport, were assessed using standard HPTS fluorescence assays (Figure S28, $\mathrm{S} 29){ }^{24,25}$ Indeed, when tested with $\mathrm{MCl}$ on external buffer, the H1-H6 channels do not present a dose-response-type activity, showing constant near to zero activity behaviors, independently of low (10 mM, Figure S28) or high $(80 \mathrm{mM}$, Figure S29) concentration of active channels in the membrane. It is very clear that $\mathrm{M}^{+} / \mathrm{H}^{+}$antiport conductance states of the assembled channels are not observed for the all studied concentrations.

We were then interested in supplementary evidence on the anion translocation mechanisms through the H-bonded hydroxylic networks, already used as channel recognition motifs for anion/proton co-transport. ${ }^{26,27}$ In order to check the anion transport activity resulting from anion/chloride antiport or anion $/ \mathrm{H}^{+}$co-transport, we conducted the HPTS assays. Different sodium salts $\left(\mathrm{Br}^{-}, \mathrm{I}^{-}, \mathrm{HCO}_{3}{ }^{-} \mathrm{CO}_{3}{ }^{2-}, \mathrm{NO}_{3}{ }^{-}, \mathrm{SO}_{4}{ }^{2-}\right)$ were used to determine the anion transport activity of $\mathbf{H 1 - H 6 ~ O H}-$ channels. On this basis, the resulting transport profiles showed rather weak near to zero transport rates for the different $\mathrm{NaX}$ salts in the presence of $10 \mathrm{mM}$ (Figure S30) and $80 \mathrm{mM}$ (Figure S31) of H1-H6 OH-channels, indicating that the transport of the hydrated anions is not governed by the solvation free energy of the anions. (Figure S31). Compounds H1 and H6, showing low water transport activities, present very low $(<5 \%)$ transport activity; most probably related to a $\mathrm{Cl}^{-} / \mathrm{X}^{-}\left(\mathrm{X}^{-}\right.$ $=\mathrm{Br}^{-}, \mathrm{I}^{-}, \mathrm{NO}_{3}{ }^{-}, \mathrm{SO}_{4}{ }^{2-}$ ) antiport. In order to verify if the $\mathrm{H}^{+}$ transport is the rate-limiting step for $\mathrm{M}^{+} / \mathrm{H}^{+}$antiport activity, we conducted HPTS assays with compounds H1-H6 in the presence of carbonyl cyanide-4-(trifluoromethoxy)phenylhydrazone, FCCP as a $\mathrm{H}^{+}$carrier (Figure S29). ${ }^{25}$ These experiments have not shown a difference with the previous results. All tested $\mathrm{OH}$-channels are totally inactive for cation transport in the presence or the absence of FCCP proton carrier. Moreover, we were interested in further testing the compounds specificity by testing them for proton transport. We thus used a classical HPTS assay and control valinomycin $\left(\mathrm{K}^{+}\right.$ cations) and FCCP (proton) transporters, known to generate a strong $\mathrm{K}^{+} / \mathrm{H}^{+}$electrogenic antiport (Figure $4 \mathrm{c}$, d). Potential proton transport acceleration is unlikely given in the case of coupling of valinomycin with H1-H6, suggesting very low proton transport rates through $\mathrm{OH}$ channels. 
a)
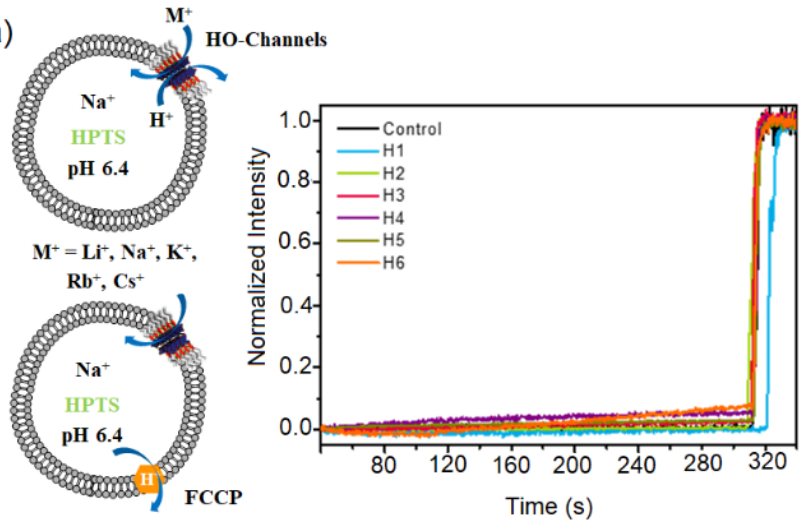

b)

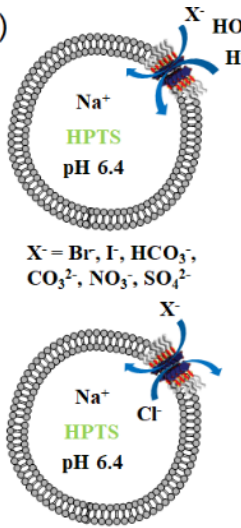

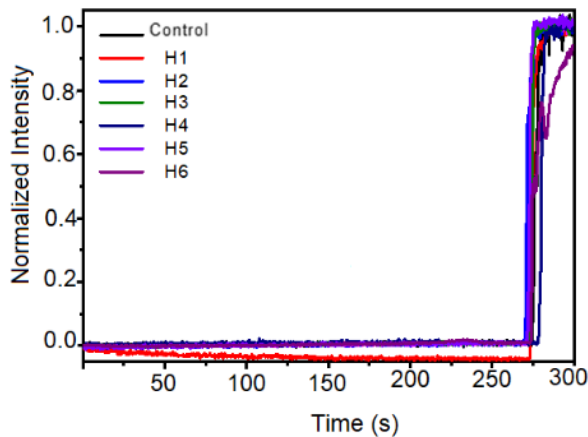
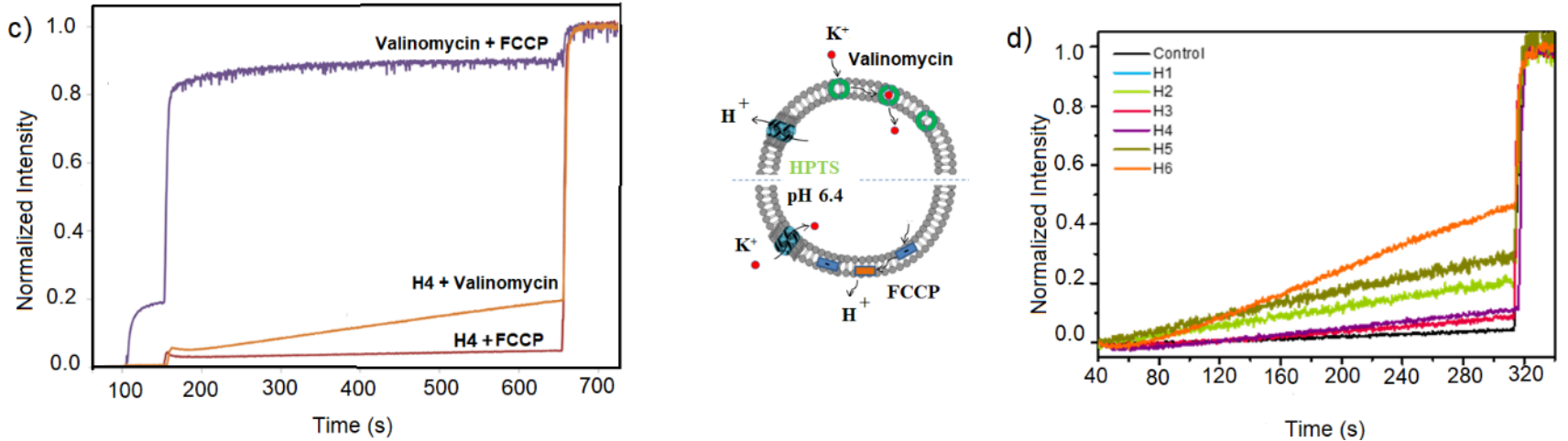

Figure 4. a) Comparison of $\mathrm{Na}^{+}$transport activity expressed as normalized fluorescence intensity of $10 \mathrm{mM}$ channels in the extravesicular media containing $100 \mathrm{mM} \mathrm{NaCl}$ (in the absence of FCCP) together with schematic representation of antiport $\mathrm{M}^{+} / \mathrm{H}^{+}$mechanisms in the absence or with the presence of FCCP as $\mathrm{H}^{+}$carrier. b) Comparison of $\mathrm{Br}^{-}$transport activity expressed as normalized fluorescence intensity of $10 \mathrm{mM} \mathrm{H} 1-\mathrm{H} 6$ channels in the extravesicular media containing $100 \mathrm{mM} \mathrm{NaBr}$, together with schematic representation of anion/ $\mathrm{H}^{+}$ symport and $\mathrm{Cl}^{-} / \mathrm{X}^{-}$antiport mechanisms. c) Transport experiments coupling $\mathbf{H} 4$ channels with valinomycin and $\mathrm{FCCP}$ as $\mathrm{K}^{+}$and $\mathrm{H}^{+}$carriers. d) Coupled transport between valinomycin $\mathrm{K}+$ carrier $(50 \mu \mathrm{M})$ and $\mathbf{H 1}-\mathbf{H 6}$ channels $(80 \mathrm{mM})-100 \mathrm{mM} \mathrm{K}_{2} \mathrm{SO}_{4}$ in the extravesicular media.

Molecular dynamics simulations of $\mathrm{H1}$ aggregates in a fully hydrated lipid bilayer environment. Molecular dynamics simulations carried out in a membrane environment, allow us to observe a range of permeation scenarios, ranging from an almost completely sealed membrane with only minimal transport at the compound/lipid boundaries, through a previously described sponge-resembling regime, ${ }^{18}$ when trying to build putative permeant channels to water-filled channel transport when stabilizing ceramide-pore-inspired hypothetical constructs through a biasing potential (Figure 5).

First, we tested crystal patches in the membrane. Depending on the simulation setup, we observed either dissolution of the crystal structure aggregate to the lipid-water interface or an impenetrable stable membrane-inserted assembly. The strong inter-molecular hydrogen bonding pattern observed in the crystal structure precluded the formation of any water wires or water clusters within the structure, suggesting that a significant re-arrangement of the compounds is required to enable water permeation. Only residual water leakage at the boundary between the crystal patch and the lipids was observed. The crystal structure is stable and displays low permeability as shown in the plot on the bottom. Fitting a line (Figure 5a, red line) to the central part of the curve leads to an estimated permeability of $4.10^{6} \mathrm{H}_{2} \mathrm{O} / \mathrm{channel} / \mathrm{s}$ (see supplementary information for details on simulation crys_gaff).
Next, we attempted the activation of the crystal patch using extensive enhanced sampling simulations to favor internal rearrangements. None of these simulations gave rise to water transport. In addition to these membrane simulations, we attempted to soak crystal patches in aqueous solution in order to assess how they might accommodate water. Despite an initial separation of the $\mathbf{H 1}$ layers by ca. $2 \AA$, the crystal patches resisted to any significant water penetration.

Further molecular simulations allowed us to assess whether the water-free crystal superstructures may adaptively reassemble within the membrane to accommodate any water translocation. These simulation results taken together suggest that the permeant membrane-embedded channel structures are substantially differing from the crystal arrangement. At some stage the compounds may re-arrange into a sponge like form, possibly a stable channel-like form, to give rise to a steady water flow. Three permeation regimes can be distinguished by fitting green, red and orange lines (Figure $5 \mathrm{~b}$ ), with respectively $3.10^{7}, 8.10^{8}$ and $1.10^{7} \mathrm{H}_{2} \mathrm{O} /$ channel/s permeability (see supplementary information for details on simulation crys_cgen). These regimes account for the crystal-like, the sponge-like and the dissolved form of the channels, which are probably most present at low concentration of channels in the membrane. 
a)
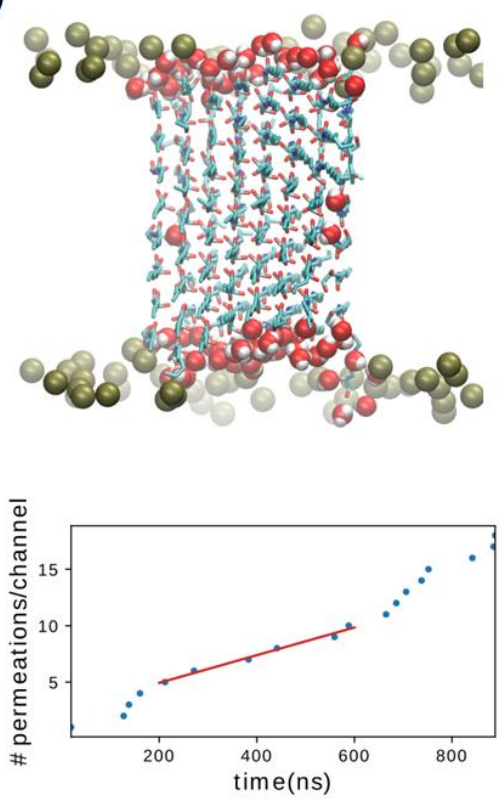

b)
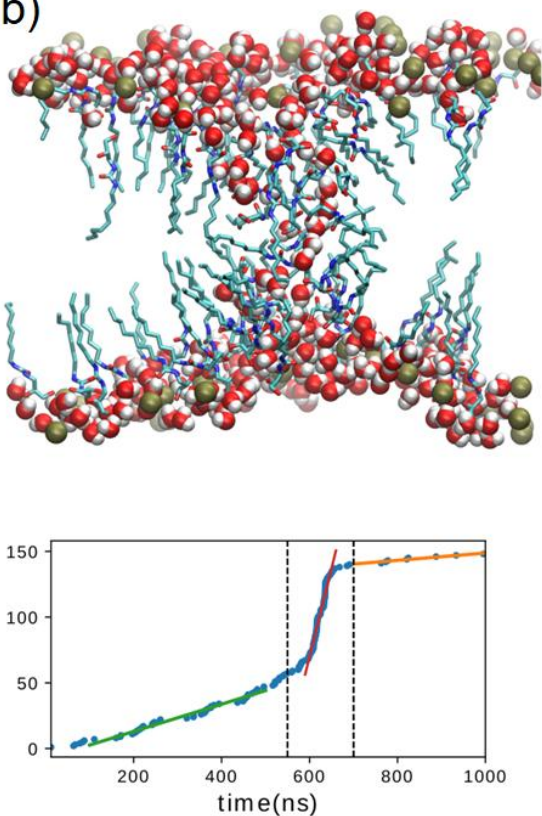

c)
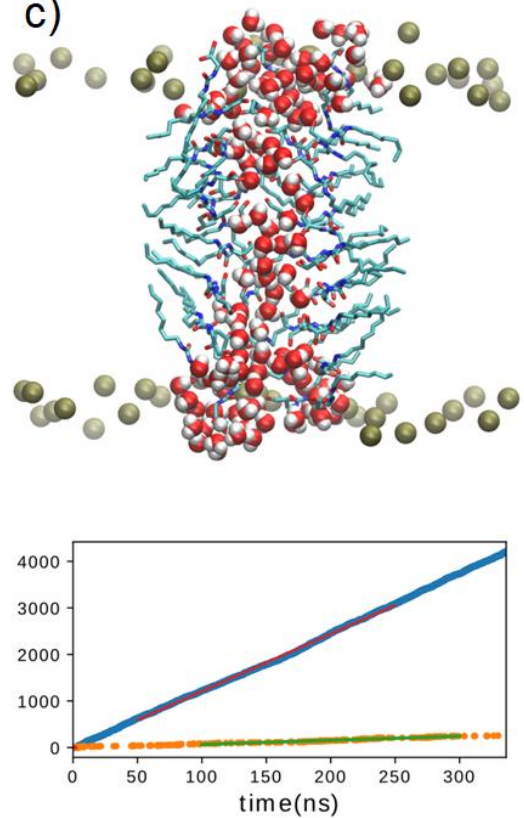

Figure 5. Molecular dynamics simulation results for $\mathbf{H 1}$ hydroxy channels in a fully hydrated lipid bilayer environment. The top row shows snapshots for three transport regimes that were observed: a) a crystal-like patch arrangement after $t=475 \mathrm{~ns}$. b) sponge-like structure after $\mathrm{t}=635 \mathrm{~ns} \mathrm{c})$ cylinder-like single membrane-spanning channel in an artificially restrained simulation with a constant force of 1000 $\mathrm{kJ} / \mathrm{mol}$ (blue, simulation harm1000) and $10 \mathrm{~kJ} / \mathrm{mol}$ (orange, simulation harm10) at $\mathrm{t}=337 \mathrm{~ns}$. Channel molecules are drawn in licorice representation. For clarity, lipid and water molecules in the bulk have been omitted. Water molecules within the lipid bilayer or close to the headgroups in contact with $\mathrm{H} 1$ are shown in van der Waals representation.

These observations may likely echo the experimental measurements, indicating a first occurrence of water permeation at low concentrations, a minimal transport regime, originating in the perturbation of the membrane arrangement induced by the presence of the $\mathrm{OH}$-channels in the lipid membrane. It is important to note that the dynamics of different channel-like forms vary significantly, as our hypothetical constructs require artificial stabilization to remain intact within the membrane.

Finally, we constructed a cylindrical pore of $\mathbf{H 1 ~ O H}-$ channels resembling the ceramides. ${ }^{22}$ Our tentative cylindrical channel pore was artificially stabilized in the simulations using harmonic restraints. The corresponding geometry is described in the supplementary information. With such a stabilized cylindrical channel, water transport is indeed observed and can be tuned by increasing the bias from $4 \times 10^{8} \mathrm{H}_{2} \mathrm{O} / \mathrm{channel} / \mathrm{s}$ (weaker force shown in orange in Figure $5 \mathrm{c}$ ) up to ca. $6 \times 10^{9}$ waters per channel per second (strong force shown in blue in Figure 5c). These observations point to the very subtle balance of interactions that needs to be achieved between the intercompound and compound-lipid/compound-water interactions to generate high permeable channels as observed at high concentrations of H1-H6 in the bilayer membrane.

This ensemble of molecular dynamics simulations was further used to check for ion permeation events. Interestingly, in none of the simulations in a bilayer environment did we observe ion permeation during the average 500 ns duration. The limiting case of a simulation with a channel that is forced to stay wide open through strong biasing forces of $1000 \mathrm{~kJ} / \mathrm{mol}$ lead to some ion permeation. As soon as this restraint force was lowered and the structure gained flexibility, ion permeation stopped.
From a structural point of view, to better understand the membrane modifications operated by this series of compounds, complementary computer simulations are required. These calculations should aim to extend our initial tests with cylindrical pores. The goal is to design and test new constructs of the OH-channel compounds with the challenge to render their architectures sufficiently stable in the membrane environment competing for interactions. So far, we were able to test some artificially stabilized constructs of cylindrical shape, which did feature permeation. However, by releasing the stabilizing bias, they were torn apart by the favorable lipid interactions in the membrane environment. Our working hypothesis for future tests is to start from the ureic core to assemble the $\mathrm{OH}$-channel compounds, in a similar fashion as has been observed for ceramide-like aggregates of cylindrical shapes ${ }^{22}$

\section{Discussion}

We show that systematic design of the octyl-ureido-polyols, lead to highly effective and selective Artificial water channels. Simple use of the variable number and sterically different hydroxyl binding sites led to unexpected increased watertransport activities at high concentration of the channels within membranes. The formation of $\mathrm{OH}$-decorated pores are probably the only common structural behaviors with ceramides that form hydrophilic pores with variable geometries. They are related to adaptive changes in the structure of the channels, reminiscent with the formation of larger pores for translocation, showing a 10-fold increase, as observed for natural ceramide transporters.

From a mechanistic point of view, the H-bonding network offered by the hydroxyl $\mathrm{OH}$ tetrads can act itself as a recogni- 
tion-based barrier within highly selective channels for water translocation. The energy penalty for binding either cations or anions would be too high, as either one of the ions can bind either the $\mathrm{O}$ or the $\mathrm{H}$ at one instance. But on the other hand, an $\mathrm{H}$-bonding water molecule can intervene with both donor and acceptor H-bonds, satisfying the full number of hydrogen bonds within the networks, making possible its facilitated translocation through the gate. The hydroxyl rich cavity of the channels can offer complete amphoteric donor-acceptor hydrogen bonding for the water, while the molecules retain a bulk like bonding environment under confined conditions of the channels. The $\mathrm{OH}$ channel architectures are very intriguing systems. It is very tempting to equate that impermeable crystalline phases or medium permeable sponge like or high permeable cylindric constructs are stabilized in the bilayer membrane. The unifying assumption is that dynamic H-bonding and hydrophobic interactions would lead to the dominant structures in a membrane environment depending on concentration of the channels and providing pathways for water translocation through the transient water clusters. The water cluster stabilization via $\mathrm{H}$-bonding to hydroxyls, confer to these channels self-adaptive behaviors, that make the intrinsic features of water sponges or clusters, particularly self-protective against ions. Having all $\mathrm{H}$ bonds saturated via the formation of water wires or water clusters, the cation/anion insertion will impede and interrupt the communication between adjacent water and $\mathrm{OH}$-channels/ pores, explaining the very low ion permeation events. I-quartet single water wires ${ }^{17}$ or sponge channels, $^{18} \mathrm{PAH}[4]$ channels $^{12}$ or porous organic cages $(\mathrm{POCs})^{14}$ are featuring this rather unique enhanced permeability of water clustering conduction systems. This strategy recently described by us, predicts almost two order of magnitude higher permeability of water clusters than of water wires channels. $^{18}$

\section{Conclusions}

We have demonstrated here that self-assembled adaptive $\mathrm{OH}$-channels can be obtained from simple octyl-ureido-polyol building blocks that present enhanced selective water permeability with almost total ion-rejection across a lipid bilayer. Slight modifications to the number and disposition of hydroxyl-OH functional groups surrounding the hydrophilic cavity of the channels or the increase of the concentration of the compounds in the membrane can drastically change the water permeability. Even with the highest margins of errors, these channels are capable of transporting water at a rate that is one order of magnitude below the natural AQP. More importantly, salt rejection and the extremely low proton permeability of the $\mathrm{OH}$-channels, offer the closest functional mimic of the natural AQP through a synthetic scaffold (Figure 6).

The present studies are of tremendous importance showing adaptive behaviors of waters inside the channels depending onthe dimension of channels determining the waterconduction behaviors as a function of concentration of $\mathrm{OH}$ channels within membranes.

It is very well known that binding energies define the flux of solutes through an artificial channel. Multiple interactions of molecules with the walls of a channel will impede permeation and result in a reduced flow rate. It was demonstrated that high water permeability values of CNTP, PAP5, PAH[4], POCs are attributed to the no-slip translocation mechanism, which is in direct correlation with the weakness of interactions of the water molecules with the walls of the nanotube.

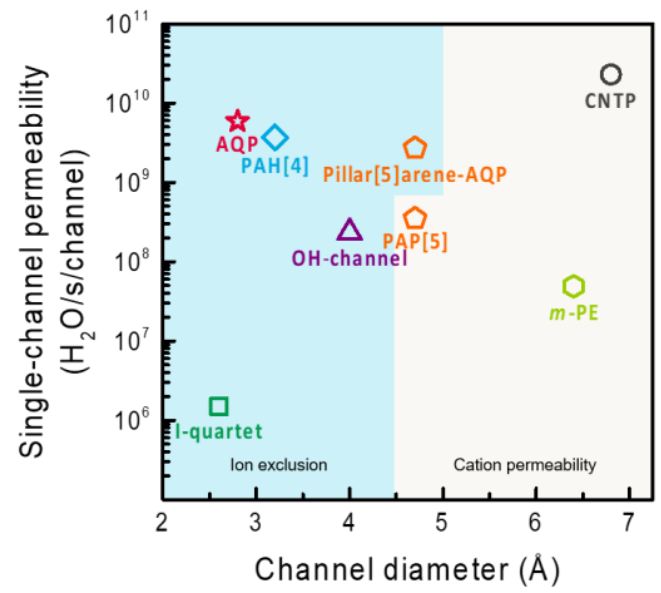

Figure 6. Comparison of single-channel permeabilities vs diameter of AQPs, CTNP and artificial water channels $(\mathrm{OH}$-channel (represented as mean value $4 \pm 2.5 \AA$ ), I-quartet, Pillar[5]areneAQP, PAH[4], PAP5, $m$-PE).

This phenomenon gives hydrophobic channels an increase in performance from the point of view of mass transfer. On the other hand, the advantage of hydrophilic channels comes from the very interactional nature of the channel cavity. Having a variety of binding moieties to interact with the transported water, specific binding motifs can be designed. This feature can be exploited, making it possible to design permselectivity filters or tailor the transport mechanism of the channels. They can adapt transient water wires and sponges which are ion selective and water permeable. Within this context this discovery of $\mathrm{OH}$-channels reported here opens up new directions and perspectives in AWCs research, ${ }^{16}$ towards the construction of selective membranes for desalination channels, ${ }^{28}$ biomimicking the functions of natural channels. ${ }^{1}$

\section{ASSOCIATED CONTENT}

Supporting Information: Materials and methods, NMR and ESI MS spectra and thermogravimetric analysis of studied compounds, X-ray crystal data, transmembrane transport experimental details, molecular dynamics simulations details. The Supporting Information is available free of charge on the ACS Publications website

\section{AUTHOR INFORMATION}

\section{Corresponding Author}

*mihail-dumitru.barboiu@umontpellier.fr

\section{Notes}

The authors declare no competing financial interest.

\section{ACKNOWLEDGMENT}

This work was supported by Agence Nationale de la Recherche ANR-18-CE06-0004-02, WATERCHANNELS. L.-B. H. wishes to thank the China Scholarship Council for the financial support. We thank Pierre Fertey for help with the data collection for $\mathrm{H} 2$ carried out at the CRISTAL beamline of the SOLEIL synchrotron 
in Saint-Aubin, France, under the ЯECIPROCS BAG proposal no. 20180913. We thank Dan Dumitrescu for the data collection for $\mathrm{H} 4$ performed on the XRD1 beamline of the Elettra synchrotron, Basovizza, Italy. The study was further supported by the "Initiative d'Excellence" program from the French State (Grant "DYNAMO", ANR-11-LABX- 0011 and grant "CACSICE", ANR-11-EQPX-0008). A.H. and M.B. thank Sesame Ile-deFrance for co-funding a display wall used for data analysis. Computational work was performed using HPC resources from GENCI (grant number A0070701714) and from LBT-HPC thanks to support from Geoffrey Letessier.

\section{REFERENCES}

(1) Barboiu, M.; Gilles, A. From natural to bio-assisted and biomimetic artificial water channel systems Acc. Chem. Res. 2013, 46, 2814-2823;

(2) Kocsis, I.; Sun, Z.; Legrand, Y. M.; Barboiu, M. Artificial water channels-deconvolution of natural Aquaporins through synthetic design. npj Clean Water 2018, 1, 13.

(3) Artificial Water Channels: Faraday Discuss. 209, The Royal Society Of Chemistry, 2019.

(4) Leduc, Y.; Michau, M.; Gilles, A.; Gence, V.; Legrand, Y. M. van der Lee, A.; Tingry, S.; Barboiu, M. Imidazole-Quartet Water and Proton Dipolar Channels. Angew. Chem. Int. Ed. 2011, 50, 1136611372.

(5) Barboiu, M. Artificial water channels. Angew. Chem. Int. Ed. 2012, 51, 11674-11676.

(7) Hu, X. B.; Chen, Z.; Tang, G.; Hou, J. L.; Li, Z. T. SingleMolecular Artificial Transmembrane Water Channels. J. Am. Chem. Soc. 2012, 134, 8384-8387.

(8) Tunuguntla, R. H.; Henley, R. Y.; Yao, Y. C.; Pham, T. A.; Wanunu, M.; Noy, A. Enhanced water permeability and tunable ion selectivity in subnanometer carbon nanotube porins. Science 2017, 357, 792-796.

(9) Li, Y.H.; Li, Z.-W.; Aydin, F.; Quan, J.; Chen, X.; Yao, Y.-C.; Zhan, C.; Chen, Y.; Pham, T. A.; Noy, A. Water-ion permselectivity of narrow-diameter carbon nanotubes. Sci. Adv. 2020, 6, eaba9966.

(10) Chen, L.; Si, W.; Zhang, L.; Tang, G. Li, Z.-T. Hou, J.-L. Chiral selective transmembrane transport of amino acids through artificial channels. J. Am. Chem. Soc. 2013, 135, 2152-2155.

(11) Shen, Y. X.; Si, W.; Erbakan, M.; Decker, K.; De Zorzi, R.; Saboe, P. O.; Kang, Y. J.; Majd, S.; Butler, P. J.; Walz, T.; Aksimentiev, A.; Hou, J. L.; Kumar, M. Highly permeable artificial water channels that can self-assemble into two-dimensional arrays Proc. Natl. Acad. Sci. U. S. A. 2015, 112, 9810-9815;

(12) Song, W.; H. Joshi, R. Chowdhury, J. S. Najem, Y. X. Shen, C. Lang, C. B. Henderson, Y. M. Tu, M. Farell, M. E. Pitz, C. D. Maranas, P. S. Cremer, R. J. Hickey, S. A. Sarles, J. L. Hou, A. Aksimentiev, M. Kumar, Artificial water channels enable fast and selective water permeation through water-wire networks. Nat. Nanotechnol. 2020, 15, 73-79.

(13) Shen, J.; Ye, R.J.; Romanies, A.; Roy, A.; Chen, F.; Ren, C.L.; Liu, Z.; Zeng, H. Q. Aquafoldmer-Based Aquaporin-like Synthetic Water Channel. J. Am. Chem. Soc. 2020, 142, 10050-10058

(14) Yuan, Y. D. Dong, J.-Q.; Liu, J.; Zhao, D.; Wu, H.; Zhou, W.; Gan, H. X.; Tong, Y. W.; Jiang, J.; Zhao, D. Porous organic cages as synthetic water channels. Nat Commun. 2020, 11, 4927.
(15) Yan, Z-J.; Wang, D.D.; Ye, Z.; Fan, T.; Wu, G.; Deng, L.; Yang, L.; Li, B.-X. Liu, J.; Ma, T.; Dong, C.; Li, Z.-T.; Xiao, L.; Wang, Y. F.; Wang, W.; Hou, J. L. Artificial Aquaporin That Restores Wound Healing of Impaired Cells. J. Am. Chem. Soc. 2020, 142, 1563815643.

(16) Huang, L.-B.; Di Vincenzo; M.; Li, Y.; Barboiu M. Artificial Water Channels-towards Biomimetic Membranes for Desalination. Chem. Eur. J., 2020, http://dx.doi.org/10.1002/chem.202003470

(17) Licsandru, E.; Kocsis, I.; Shen, Y. X.; Murail, S.; Legrand, Y. M.; van der Lee, A.; Tsai, D.; Baaden, M.; Kumar, M.; Barboiu, M. Salt-Excluding Artificial Water Channels Exhibiting Enhanced Dipolar Water and Proton Translocation. J. Am. Chem. Soc. 2016, 138, 5403-5409.

(18) Murail, S.; Vasiliu, T.; Neamtu, A.; Barboiu, M.; Sterpone F.; Baaden, M. Water permeation across artificial I-quartet membrane channels: from structure to chaos. Faraday Discuss., 2018, 209, 125 148.

(19) Di Vincenzo, M.; Tiraferri, A.; Musteata, V.-E.; Chisca, S.; Sougrat, R.; Huang, L.B. Nunes, S. P.; Barboiu, M. Biomimetic artificial water channels membranes for enhanced desalination. Nature Nanotech. 2020, DOI:10.1038/s41565-020-00796-x.

(20) Wiegandt, H.; Glycosphingolipids, in Advances in Lipid Research, R. Paoletti, D. Kritchevsky (Eds.), Academic Press, New York, 1971, pp. 249-289.

(21) Kanfer, J. N.; Sphingolipid Metabolism, in: Sphingolipid Biochemistry, Kanfer, J. N.; Hakomori S. (Eds.), Plenum Press, New York, 1983, pp. 167-247

(22) Siskind L. J.; Colombini, M.; The Lipids C2- and C16-Ceramide Form Large Stable Channels. J. Biol. Chem. 2000, 275, 38640-38644.

(23) Ferdanic, R.; Lib, R.; Pajewskic, R.; Pajewskac, J.; Wintera, R. K.; Gokel G. W. Transport of chloride and carboxyfluorescein through phospholipid vesicle membranes by heptapeptide amphiphiles. Org Biomol Chem. 2007, 5, 2423-2432.

(24) Matile, S.; Sakai, N. in Analytical Methods in Supramolecular Chemistry, Schalley, C. A., Ed. Wiley -VCH: Weinheim, pp 381-418, 2007.

(25) Li, Y.-H.; Zheng, S.-P.; Legrand, Y.-M.; Gilles, A.; Van der Lee A.; Barboiu M. Structure-driven selection of adaptive transmembrane $\mathrm{Na}^{+}$carriers or $\mathrm{K}^{+}$channels. Angew. Chem. Int. Ed. 2018, 57, $10520-$ 10524

(26) Saha, T.; Dasari, S.; Tewari, D.; Prathap, A.; Sureshan, K. M.; Bera, A. K.; Mukherjee, A.; Talukdar, P. Hopping-Mediated Anion Transport through a Mannitol-Based Rosette Ion Channel. J. Am. Chem. Soc. 2014, 136, 14128-14135.

(27) Saha, T.; Gautam, A; Mukherjee, A.; Lahiri, M.; Talukdar, P. Chloride Transport through Supramolecular Barrel-Rosette Ion Channels: Lipophilic Control and Apoptosis-Inducing Activity. J. Am. Chem. Soc. 2016, 138, 16443-16451.

(28) Di Vincenzo, M.; Tiraferri, A.; Musteata, V.-E.; Chisca, S.; Sougrat, R.; Huang, L.-B.; Nunes, S. P.; Barboiu, M. Biomimetic artificial water channels membranes for enhanced desalination. Nat. Nanotechnol. 2020, DOI: 10.1038/s41565-020-00796-x. 


\section{Graphical abstract}

Networks of $(\mathrm{OH}---\mathrm{O})_{4} \mathrm{H}$-bonding quartets for the construction of adaptive water channels

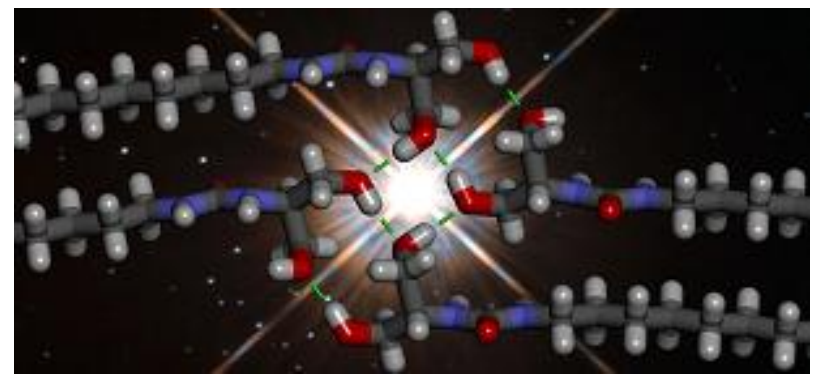

